\title{
Ayudas en línea para lectura multimodal en lengua extranjera
}

\author{
John Jiménez \\ Universidad de Antioquia, Medellín, Colombia
}

Artículo recibido el 11 de enero de 2013, Versión final recibida el 29 de mayo de 2013)

Este artículo describe un estudio ${ }^{1}$ con un enfoque mixto, en el que estudiantes universitarios de inglés como lengua extranjera (EFL), utilizaron ayudas en línea - tales como diccionario, traductor, síntesis, entre otras - como estrategias para facilitar a otros estudiantes, con nivel de principiantes en inglés, la lectura y comprensión de textos multimodales en este idioma. Se recolectó información cuantitativa y cualitativa a través de la interfaz de la plataforma virtual, de cuestionarios semi-estructurados y de entrevistas. Los resultados muestran que el traductor y el diccionario en línea, fueron las ayudas más utilizadas, al mismo tiempo que permitieron mejorar la comprensión de los textos multimodales. Finalmente, más de la mitad de los estudiantes de ambos grupos prefirieron combinar el uso de textos multimodales con textos impresos, y la mayoría de estudiantes estuvieron de acuerdo en sugerir el uso de textos multimodales en los cursos regulares de competencia lectora en inglés.

\section{Introducción}

En muchos contextos donde se aprende una lengua extranjera, buena parte del conocimiento de los estudiantes surge como resultado de la enseñanza directa en el aula utilizando medios tradicionales como la información impresa, y la pizarra, entre otros. Sin embargo, el conocimiento también puede ser propiciado en estos y otros escenarios gracias a la mediación de las Tecnologías de la Información y la Comunicación (TIC).

Según Knobel, Coiro, Leu y Lankshear (2008), más de mil millones de lectores están en línea cada día desde sus hogares, lugares de trabajo y escuelas, hecho que soporta la importancia del uso de Internet en diferentes contextos, incluyendo el educativo. En este sentido, Coiro (2005), Leu (2002) y Huang, Chern y Lin (2009) reconocen que Internet tiene hoy más acogida en la enseñanza de una Lengua Extranjera (LE) que los libros, la televisión o cualquier otro medio, porque esta red se ha convertido en el principal proveedor del material que se utiliza en el aula.

En el ámbito internacional, algunos estudios de investigadores como Anderson (2003), Huang, Chern y Lin (2009), y Akyel y Ercetin (2009), han desarrollado 
temáticas relacionadas con el uso de estrategias para la comprensión de textos multimodales. Asimismo, Rubin, Chamot, Harris y Anderson (2007) han demostrado la pertinencia de la enseñanza de estrategias en el desarrollo de la comprensión lectora en una lengua extranjera (LE). Diferentes áreas como la psicología educativa, la lingüística aplicada, la sociolinguiística, la didáctica, la antropología, la sociología y la comunicación se han interesado en investigaciones complejas del papel del idioma, la alfabetización y el aprendizaje, especialmente en el ámbito educativo en un mundo dominado por los avances tecnológicos. También en el ámbito educativo, la necesidad de un nuevo tipo de ciudadano, diferente y alfabetizado es vox populi en el discurso público, es decir, un ciudadano que tenga habilidades para leer y escribir un e-mail, hipertextos, encontrar información útil en la web, entre otras.

Con los resultados de este proyecto se pretende incentivar en los docentes el uso de ambientes multimodales como un escenario de aprendizaje complementario al ofrecido en el aula tradicional, en coherencia con los desarrollos tecnológicos que constantemente se incorporan a la vida diaria de los estudiantes, y los cuales, en el contexto educativo, se derivan en el aprovechamiento de las posibilidades didácticas de las TIC.

\section{Estado de la cuestión}

En esta sección se mencionan algunos de los fundamentos teóricos en los que se sustenta este proyecto en general, las TIC como instrumentos de aprendizaje de una LE e investigaciones relacionadas con el presente estudio. En primer lugar, se discute la investigación en la enseñanza de estrategias, tanto de lectura en lengua primera como para el aprendizaje de lenguas extranjeras.

Para Grabe y Stoller (2002), un estrategia es una guía para la acción, en el sentido que busca orientar al lector para la obtención de ciertos resultados. La estrategia da sentido de coordinación a todo lo que se hace para alcanzar una meta. Según Anderson (2003), el uso de estrategias apunta a que los estudiantes mejoren en el desempeño de la lengua extranjera, en este sentido Anderson (2003) define las estrategias como acciones conscientes que los estudiantes realizan para mejorar su aprendizaje del idioma. Para efectos de este proyecto, el concepto de estrategia se define como habilidad o técnica utilizada por los lectores para el desarrollo de actividades de lectura. 
A continuación se hace un breve recuento de las investigaciones que presentan algunos aspectos similares a los del presente proyecto. Al-Seghayer (2001) utilizó la multimedia para la enseñanza de una segunda lengua (SL) para aprender vocabulario, entre las ayudas que tenían a disposición los estudiantes estaban glosas, gráficas, video y sonido. Por su parte, Sakar y Ercetin (2005) han examinado temas como anotaciones para la lectura de textos hipermediales en FL con texto, audio, video, anotaciones y pronunciación. En nuestro caso el listening, speed reading, y key words tuvieron un porcentaje de uso importante. Stakhnevich (2002) ha explorado procesos de lectura de estudiantes de SL respecto al uso de herramientas en la lectura multimodal que incluían glosas, definiciones, animaciones, diccionarios entre otras.

Diferentes investigadores han incursionado en el campo de las estrategias para leer textos multimodales, entre ellos tenemos a Plass y Jones (2005) quienes presentan un escenario para la lectura en inglés en LE, por medio del uso del video y el acceso a hipervínculos, traductores, oraciones descriptivas, información previa y guías de pronunciación. Mazzali-Lurati y Schulz (2003), con transposiciones hipertextuales que incluían texto, imagen, video y audio.

Zhang y Duke (2008) describen un estudio sobre estrategias para el desarrollo de tareas con propósitos diferentes, y de manera similar, Huang, Chern y Lin (2009), investigan el uso de estrategias por parte de estudiantes de EFL. En el estudio de éstas investigadoras, las estrategias que más altos porcentajes obtuvieron en su investigación fueron translator $27.3 \%$, dictionary $19.19 \%$ y highlight con $10.53 \%$. De igual manera, Anderson (2003) ha investigado acerca del uso de estrategias en línea por estudiantes de ESL y EFL, y las más utilizadas fueron estrategias para la solución de problemas como relectura de textos difíciles, hacer pausa para pensar en lo que se lee entre otras, y Akyel y Ercetin (2009) han examinado las estrategias para la lectura en formato hipermedial entre las que se contaba con glosario interno, información general y previa sobre el tema, texto, audio, gráficas y video.

En el presente estudio se han aplicado parámetros similares de los estudios mencionados, aunque los diferentes estudios fueron desarrollados en diversos contextos, se puede resaltar el uso de herramientas tecnológicas como recursos de apoyo para la enseñanza y el aprendizaje del inglés como LE. 


\section{Contexto del estudio}

Participantes. Este proyecto se desarrolló con dos grupos de estudiantes universitarios matriculados en los cursos de Competencia Lectora en Inglés 1, que fueron ofrecidos por la Sección de Servicios de la Escuela de Idiomas de la Universidad de Antioquia, en el segundo semestre de 2011 en la sede de Medellín, Colombia.

La muestra estuvo conformada por dos grupos de estudiantes de la Facultad Nacional de Salud Pública de la misma universidad, que cursaban diferentes programas de la mencionada facultad y que comparten cursos como este de inglés. El grupo uno, fue compuesto por 17 estudiantes, y el grupo dos fue constituido por 15 estudiantes, para un total de 32 participantes de los programas de Administración en Servicios de Salud, Administración en Salud Sanitaria y Ambiental, y Gerencia en Sistemas de Información en Salud.

Los estudiantes participantes en el estudio estaban matriculados, en su mayoría, en el cuarto, quinto, sexto y séptimo semestre de sus programas de estudio. Respecto al nivel de inglés de los estudiantes participantes en el proyecto, la muestra de estudiantes se clasifica como beginners, lo que se evidencia en el tipo de curso y nivel de inglés en el que se encontraban matriculados.

Este estudio tiene como objetivo contribuir a la cualificación de los ambientes de enseñanza-aprendizaje del inglés como lengua extranjera a partir de la implementación de estrategias para la lectura de textos en un contexto multimodal. Con esta finalidad, se procura dar respuesta a las siguientes preguntas:

1. ¿Cuáles son las estrategias para la lectura de textos multimodales que más emplean estudiantes universitarios de inglés como lengua extranjera?

2. ¿Cuál es la relación que existe entre el tipo de estrategias empleadas para la lectura de textos multimodales y el nivel de comprensión lectora alcanzado por estudiantes universitarios de inglés como lengua extranjera?

3. ¿Cómo afectan las estrategias para la lectura de textos multimodales el proceso de comprensión lectora de los estudiantes universitarios de inglés como lengua extranjera? 


\section{Metodología}

Para dar respuesta a las preguntas que se plantearon en este estudio se utilizó un enfoque mixto, que como argumentan Hernández, Fernández, y Baptista (2004), posibilita la integración de los enfoques cualitativos y cuantitativos durante las diferentes fases del proceso de investigación. Una manera de describir el método mixto de investigación, de acuerdo con Dörnyei (2007), es la combinación de métodos cualitativos y cuantitativos en un mismo proyecto de investigación. La variedad en sus posibles combinaciones es muy rica, va más allá de un orden simplemente secuencial (por ejemplo a una fase de un proyecto le sigue otra con otro enfoque). Más que eso, los principios cuantitativos y cualitativos pueden ser combinados en la fase de análisis de datos, cuantificándolos o cualificándolos.

Dörnyei también dice que el método mixto no necesariamente implica una distribución igual en la recolección o análisis de datos cuantitativos y cualitativos. Este estudio utilizo un enfoque principalmente cuantitativo y se utilizaron datos cualitativos con el propósito de hacer una triangulación de datos.

\section{Recogida de datos: instrumentos y contribución al análisis}

Para la recolección de los datos cuantitativos se utilizó la sección de lectura de Computer-Based TOEFL Test, CD-ROM for Computer-Based Exam (Gear y Gear, 2002), el cual se les administró a los estudiantes antes de iniciar la intervención y después de finalizada ésta. Con éste se pretendía saber si había habido algún avance en los estudiantes a nivel de comprensión que se le pudiera atribuir a la intervención.

Se recolectaron datos cualitativos para el proyecto por medio de cuestionarios semi-estructurados, que los estudiantes diligenciaron después de interactuar con las lecturas multimodales. En éstos se indagaba por el uso de las ayudas que tenían a disposición también se les pidió a los estudiantes que explicaran el porqué de sus respuestas, para así ampliar la información sobre las respuestas que habían seleccionado.

Otros datos cualitativos también se obtuvieron por medio de entrevistas que se realizaron a estudiantes que alcanzaron un alto o bajo desempeño en los test de comprensión de cada lectura. El propósito de las entrevistas era conocer la percepción de los estudiantes sobre el uso de las ayudas que habían utilizado. 
Asimismo, se recogieron datos cualitativos y cuantitativos usando los registros de navegación en la interfaz de la plataforma virtual. Para este fin, los datos fueron almacenados en la medida en que los estudiantes interactuaban con cada una de las seis lecturas utilizadas y sus respectivos test de comprensión. Como paso previo a la intervención o fase experimental del estudio, se realizó un pilotaje a la multimedia, el cual permitió mejorar algunos aspectos con base en las recomendaciones de los estudiantes y al uso mismo de la interfaz.

\section{Actividades}

Selección materiales ${ }^{2}$ Para las lecturas que se utilizaron en la interfaz se seleccionaron en total seis textos. Los estudiantes disponían de dos horas para interactuar con cada lectura y desarrollar las actividades que estas proponían.

A continuación, la Tabla 1 describe la clasificación promedio que tres profesionales efectuaron con respecto al nivel de dificultad de las seis lecturas y de sus respectivos test de comprensión.

Tabla 1. Grado de dificultad de cada lectura y test de comprensión; 1(muy fácil) a 5 (muy difícil)

Lectura
Grado de Dificultad de la

Lectura
Grado de Dificultad del Respectivo Test de Comprensión

\begin{tabular}{ccc}
\hline Valentine`s day & Intermediate-Mid & 3.66 \\
\hline Immigration in Italy & Intermediate-Mid & 3.66 \\
\hline Immigration in America & Intermediate-Low & 3 \\
\hline Tsunamis & Intermediate-High & 4.33 \\
\hline Schizophrenia & Intermediate-Mid & 4 \\
\hline Overweight & Intermediate-High & 4.33 \\
\hline
\end{tabular}

Interfaz y ayudas. Para este estudio se diseño y utilizó una interfaz gráfica multimodal como herramienta didáctica. El propósito de ésta era recoger datos de los estudiantes de manera automática e individualizada, a partir de la interacción de éstos con las lecturas. Las ayudas disponibles tenían como finalidad facilitar la lectura y compresión de los textos, y fueron: preview -esta ayuda permite que el lector en un párrafo tenga una vista previa o un anticipo sobre el tema de lectura-, y keywords -con este recurso el estudiante tiene acceso a palabras claves del texto con su respectivo significado(basados en Huang, Chern y Lin, 2009 y Akyel y Ercetin, 2009). De los mismos autores, se adoptaron las ayudas summary -esta ayuda permite el acceso a un párrafo en el que se hace una síntesis del texto-, speed reading -con esta ayuda el estudiante puede ajustar el audio del texto si considera que esta muy rápido puede realizar la misma 
escucha sin que se distorsione la calidad del audio- y listening -permite que el estudiante pueda escuchar y leer simultáneamente la lectura del texto en inglés, y repetirlo las veces quiera-, ésta última ayuda fue retomada de Al-Seghayer (2001), Sakar y Ercetin (2005), y Akyel y Ercetin (2009).

De manera similar las ayudas outline -es una síntesis exhaustiva de cada uno de los párrafos del texto en un párrafo-, translator -los estudiantes tienen acceso a un traductor de inglés a español, que pueden utilizar cuando lo crean conveniente-, highlight -con este recurso el estudiante puede resaltar cualquier parte del texto frente al cual tenga dificultades de vocabulario, gramática o información que considere importante-, background knowledge -este recurso ofrece al estudiante información general sobre el tema que propone el texto-, fueron tomadas de Huang, Chern y Lin (2009) y glossary -se encuentra disponible en texto y audio, de esta manera se pueden escuchar tanto la definición como los ejemplos que se ofrecen para ilustrar la palabra en contexto, lo que le sirve al participante para la comprensión de la palabra o expresiónfue adoptada de Akyel y Ercetin (2009).

Intervención. Se les ofreció a los estudiantes participantes una sesión de dos horas semanales en un aula con computadores y conexión a Internet, en la cual cada estudiante podía utilizar un equipo. Se desarrollaron cuatro sesiones de ambientación planificadas por el profesor para explorar sitios web y lecturas en línea, luego se les proporcionó a los estudiantes un nombre de usuario y contraseña para acceder a la interfaz en la que interactuaron con seis textos multimodales en inglés.

El investigador implementó una planeación metodológica adaptada por él, basada en las etapas de un modelo de enseñanza sugeridas por Rubin, Chamot, Harris y Anderson (2007), que incluía los siguientes momentos: 1) autoconciencia, 2) modelado, 3) práctica autónoma, y 4) auto-evaluación. En el primer momento se pretendía que el estudiante tomara conciencia de las estrategias que conocía, para planear y monitorear la lectura de forma intencional.

En la primera sesión el investigador buscó efectuar un diagnóstico sobre la navegación que realizaban los estudiantes con este tipo de textos. En el segundo momento, se hace el modelado por parte del profesor, quien desarrolló el ejercicio de pensar en voz alta sobre el uso de todas las ayudas disponibles y respondió el test de comprensión. 
A partir de la tercera sesión y para las restantes tres lecturas, todos los estudiantes dispusieron de un instructivo en papel para que de forma autónoma utilizaran las ayudas, también se les recordaba la ruta de ingreso al sitio web, además de pautas para un uso efectivo de las herramientas que tenían a disposición. Y en el último momento el estudiante realizaba una autoevaluación sobre la efectividad de las ayudas que había utilizado a través del diligenciamiento de un cuestionario después de cada lectura.

\section{Técnicas de análisis de datos}

El análisis estadístico fue realizado usando el paquete estadístico SPSS para Windows versión 20.0 y el Text Analysis for Surveys 4.1. Para las variables de tipo cualitativo medidas en el proyecto, se calcularon las frecuencias, porcentajes, cuartiles, mientras que para las variables de tipo cuantitativo se calcularon las medidas de tendencia central y de dispersión correspondientes.

Para la comprobación de las hipótesis estadísticas, se calculó primero la prueba estadística no paramétrica de Kolmogórov-Smirnov (prueba K-S), al validar si las variables de interés tenían una distribución normal, se encontró que este supuesto no se cumple en todas. Igualmente, se aplicaron las pruebas no paramétricas U de Mann Whitney (U de M), Wilcoxon, McNemar en los casos que lo requirieran, y se aplicó la tStudent en las situaciones que presumían normalidad.

Para probar la confiabilidad de los instrumentos, se utilizó el Alfa de Cronbach y la consistencia interna de la escala con la correlación Ítem-Total. La validez de la escala se evaluó mediante análisis factorial. En todas las pruebas se consideró una diferencia como significativa si el p-valor era menor que el nivel de significación $\alpha=0,05$.

Para efectos de este proyecto y con el propósito de procurar una visión más holística y darle más validez al mismo, se utilizó la triangulación con el objetivo de aumentar la validez de los resultados obtenidos en una investigación y aminorar los problemas de sesgo en el análisis e interpretación de los datos (Rodríguez, 2005). Luego de la sistematización, se pasó al análisis e interpretación de los datos que fueron recolectados por medio del TOEFL, los cuestionarios, las entrevistas, y el registro de datos en la interfaz. 


\section{Resultados}

Para dar respuesta a la pregunta que indagaba por las estrategias más utilizadas por estudiantes universitarios con nivel de principiantes en inglés en las lecturas de textos multimodales en este idioma, se retoman algunos de los hallazgos que se obtuvieron después del proceso de análisis e interpretación.

En un cuestionario administrado al inicio del curso se les preguntó a los estudiantes por las ayudas que utilizaban para comprender los textos que leían en inglés, los resultados indicaron que los estudiantes preferían ayudas como el traductor $(87.5 \%)$ $\mathrm{y}$, en menor medida, el diccionario (18.8\%).

Con respecto a las herramientas más utilizadas por los estudiantes en la interacción con la interfaz, se encontró que speed reading fue la ayuda más utilizada por el grupo uno, en un promedio de tres veces por lectura. La lectura donde más se utilizó esta herramienta fue en Immigration in Italy (ver Anexo 1). También se observó cierta tendencia en el grupo uno a efectuar un uso mayor de ayudas. No obstante, no se hallaron diferencias significativas de uso entre el grupo uno y el grupo dos ( $\mathrm{p}$-valor $>0.05)$. La ayuda que los participantes del grupo dos utilizaron mayor número de veces fue highlight (cerca de 3 veces por lectura), y Tsunamis la lectura donde se dio el uso más recurrente de esta herramienta.

En relación con las ayudas más utilizadas para la lectura de textos multimodales en inglés, de acuerdo con los datos obtenidos a través de los cuestionarios, el total de los estudiantes de los grupos uno y dos expresaron haber utilizado translator en un $81 \%$, dictionary en un $42 \%$, speed reading en un $22.4 \%$, highlight en un $20.5 \%$, glossary en un $19.5 \%$, preview en un $15.6 \%$, summary en un $13.7 \%$, key words en un $13.2 \%$, background knowledge en un $12.7 \%$, listening en un $12.2 \%$ y outline en un $2.9 \%$. Los porcentajes exceden el $100 \%$ porque los estudiantes podían seleccionar más de una opción.

En las entrevistas uno de los estudiantes especificó sobre las ayudas que utilizó "el speed reading, el listening, el highlight, el translator, y el diccionario" (Entrevista: 132). Otro estudiante agregó: "Lo que fue Summary, Preview y la otra Outline, me ayudaron como a sintetizar más la idea del texto, entonces me favoreció mucho utilizar esas ayudas" (Entrevista: 2-5). Finalmente, una estudiante dijo: "el traductor me pareció importante para la comprensión" (Cuestionarios: 2-8). 
Para dar respuesta a la relación entre el tipo de estrategias empleadas para la lectura de textos multimodales y el nivel de comprensión alcanzado por estudiantes universitarios con nivel de principiantes en inglés se toman algunos de los resultados obtenidos después del análisis e interpretación de diversos datos.

Para medir la influencia de la propuesta metodológica se utilizo la sección de lectura del test TOEFL que constaba de 4 lecturas y 50 preguntas. A los dos grupos de estudiantes de comprensión lectora en inglés se les administraron dos pruebas diferentes, una al inicio y otra al final del curso, en cada grupo se realizó una prueba $t$ para las muestras. Los resultados de esta prueba mostraron que en el grupo uno, el uso de estrategias ayudó significativamente en el proceso de comprensión lectora de estos (valor-p=0.049). No obstante, este efecto no estuvo tan marcado en el grupo dos, en el cual se observó un aumento al final de la intervención metodológica, sin llegar a ser mínimamente superior al que inicialmente presentó el grupo uno.

En la sistematización, análisis e interpretación de los cuestionarios diligenciados por los estudiantes después de interactuar con cada lectura, los resultados mostraron que, dependiendo de la lectura, los estudiantes utilizaron las ayudas disponibles en diferente medida (valor-p <0.001). Tsunamis constituyó la lectura en la cual el $100 \%$ de los estudiantes reconocieron usar ayudas y Valentine's day la lectura donde menos expresaron usar las ayudas $13.9 \%$. Sin embargo, el $61 \%$ del total de estudiantes de ambos grupos hizo uso de las herramientas, sin presentar mayores diferencias entre ellos (valor-p =0.906). Al examinar por grupo el uso de las ayudas en cada una de las lecturas, tampoco se encontraron diferencias significativas (valor-p $=1.000$ ), en otras palabras ambos grupos usaron de manera similar las ayudas durante las lecturas (ver Anexo 2).

Después de la sistematización, análisis e interpretación de los datos registrados en la interfaz con relación a los test de comprensión de cada lectura, que constaba de diez preguntas de escogencia múltiple con cuatro opciones y una única respuesta correcta, se encontró que la cantidad de respuestas correctas en los test de comprensión de las lecturas no presentó diferencias en ambos grupos, presentando un promedio de 5. Ambos grupos obtuvieron más respuestas correctas en el test de comprensión de Immigration in Italy (6.2 respuestas buenas en promedio), mientras que dieron menos 
respuestas acertadas en el test de comprensión de Immigration in America (4 respuestas correctas en promedio). Estos resultados se visualizan en el Anexo 3.

De acuerdo con los datos registrados en los cuestionarios que los estudiantes diligenciaban después de cada lectura, se encontró la siguiente relación porcentual con respecto a las ayudas que les permitieron a los estudiantes, tanto del grupo uno como el grupo dos, una mejor comprensión de la lectura de textos multimodales en inglés: translator en línea alcanzó un 76.6\%, dictionary en línea un 34.1\%, listening un 18.5\%, preview un $16.1 \%$, glossary un $14.6 \%$, speed reading un $12.7 \%$ y summary un $12.7 \%$ cada una, background knowledge un $10.7 \%$, highlight un $10.2 \%$, outline un $7.3 \%$, y key words un $6.8 \%$. La suma de los porcentajes excede el $100 \%$ porque los estudiantes podían escoger más de una respuesta.

Resulta relevante el alto porcentaje de estudiantes que utilizaron el traductor (76.6\%), uno de los estudiantes justificó su elección de la siguiente manera: “[escogí] el traductor porque es fácil para resolver las dudas sobre la lectura y el glosario porque había palabras que desconocía" (Cuestionarios: 2-9). Otro estudiante del grupo dos agregó "el traductor, sí, porque habían unas palabras que, pues, no logro comprender muy bien, entonces el traductor ayuda mucho" (Entrevista: 2-3).

Para dar cuenta de la manera en que afectó el proceso de comprensión lectora el uso de estrategias por parte de estudiantes universitarios con nivel de principiantes en el idioma inglés se retoman algunos de los hallazgos.

Después de la sistematización, análisis e interpretación de los resultados del test TOEFL, las pruebas estadísticas determinaron que la cantidad de respuestas acertadas presentaban una distribución normal, tanto al inicio (p-valor=0.568) como al final (pvalor=0.439), lo cual indica la viabilidad de aplicar pruebas estadísticas paramétricas.

Los estudiantes del grupo uno, tanto al inicio como al final del TOEFL presentaron mayor número de respuestas acertadas en comparación a los estudiantes del grupo dos. No obstante, se puede afirmar que al inicio, los dos grupos presentaron un nivel se asertividad similar (valor- $\mathrm{p}=0.276$ ).

Con respecto a la distribución de la media y la desviación estándar puede decirse que la pequeña diferencia que existía en el conocimiento de inglés entre los estudiantes de ambos grupos se hace más notoria, favoreciendo a los estudiantes del grupo 1 (valor- 
$\mathrm{p}=0.042$ ), lo cual quiere decir que la metodología empleada mejoró el nivel de comprensión lectora alcanzado por los estudiantes del grupo uno.

Cabe destacar, que de las ayudas que más se utilizaron para responder las preguntas del test de comprensión, el porcentaje más alto lo obtuvo el Translator (58.5\%), seguido del Dictionary ( $18.5 \%$ ), el Summary y el Highlight (2.9\% cada una), el Background Knowledge (2\%) y, por último, el Preview (1\%).

Los estudiantes justificaron el uso de ayudas para responder el test de comprensión así: "Para responderlo, me base más en el traductor, en algunas preguntas para mirar palabras que no comprendía muy bien, entonces las traducía" (Entrevista: 23). Otro estudiante agregó "Sí [utlizaba] las mismas: traductor, diccionario y subrayado" (Entrevista: 2-22). Finalmente, otro estudiante del grupo 1 expresó: "El Translator definitivamente fue la mejor herramienta" (Cuestionarios: 1-26).

Finalmente, se puede afirmar que el uso de estrategias para la lectura de textos multimodales afectan el proceso de comprensión lectora de estudiantes universitarios con nivel de principiantes de inglés como lengua extranjera de una manera positiva, lo que se refleja en el hecho de que, el (78.5\%) de los estudiantes mostró su preferencia por la lectura de textos multimodales, justificándolo de la siguiente manera: "El multimodal ofrece más ayudas que facilitan más la comprensión de texto, mientras que los impresos no tienen herramientas que faciliten la comprensión del texto" (Entrevista: 1-9), otro estudiante agregó: “[el] multimodal, porque tiene más ayudas, más herramientas y esto nos ayuda a comprender mejor el texto" (Entrevista: 2-18).

\section{Limitaciones del estudio}

Hay dificultad para monitorear y evaluar en el nivel dos de competencia lectora en inglés para asegurar si los estudiantes han internalizado los aprendizajes que desarrollaron en el primer nivel. Igualmente, representan dificultades intentar indagar si toman el nivel dos de comprensión lectora en el siguiente semestre académico o lo dejan para semestres posteriores, o si optan por presentar el examen de suficiencia, y en caso de aprobarlo cumplir con el requisito que les exige la universidad.

Del mismo modo, es difícil saber si el hecho de que se entrevistaron los alumnos sobre el profesorado antes de finalizar el curso (los estudiantes aún no sabían si aprobaban o no) puede haber influenciado las respuestas. Otra limitación relacionada 
con el periodo académico en que se realizo la recolección de la información para este estudio, coincidió con un periodo de protestas y disturbios estudiantiles que llevó a una incertidumbre y anormalidad académica, lo que obligó a reprogramar sesiones que estaban planificadas con el grupo uno de estudiantes en el aula de computo.

Una última limitación está relacionada con el número de estudiantes de los dos grupos (17) en el uno y (15) en el dos, no permite que los resultados del presente estudio ubicándolos en el contexto en el que se desarrolló se puedan generalizar.

\section{Discusión}

Entre los hallazgos se da cuenta de que todas las ayudas de que disponían los estudiantes para la lectura de textos multimodales fueron utilizadas, y de que translator, con el $81 \%$, y dictionary, con el $42 \%$, se impusieron ante las otras ayudas, que alcanzaron porcentajes más bajos de uso. Lo que reafirma información que se había analizado sobre un cuestionario demográfico administrado a los estudiantes antes del inicio del proyecto, se encontró que para la lectura y comprensión de textos en inglés, los estudiantes utilizaban ayudas como el traductor $(87.5 \%)$ y, el diccionario en línea $(18.8 \%)$.

Hallazgos éstos que Huang, Chern y Lin (2009) ratifican en un estudio sobre el uso de estrategias utilizadas por estudiantes universitarios de inglés como lengua extranjera, en la lectura de textos en inglés en línea, quienes encontraron que el uso del diccionario y traductor en línea fueron las ayudas más utilizadas y que hacían parte de las estrategias de apoyo que tenían a disposición los estudiantes para la lectura y comprensión de este tipo de textos.

Hallazgos similares se han encontrado en un estudio realizado por Jiménez (2009) en el que estudiantes universitarios con nivel de principiantes en inglés, para la lectura y comprensión de textos en línea en este idioma utilizaron el traductor $71 \%$ y el diccionario en línea en un $43 \%$.

Otras ayudas también utilizadas en este estudio y que permitieron mejorar la comprensión de los textos multimodales en inglés fueron: preview con 15.6\%, summary $13.7 \%$ y outline 2.9\%. Como afirman Perfetti (1994) y Stanovich (2000) los lectores poco hábiles en lengua extranjera hacen un mayor uso del contexto para reconocer palabras desconocidas mientras leen. 
En las lecturas multimodales del presente estudio se incorporó la ayuda Background knowledge con un $12.7 \%$ tuvo un éxito relativo en la comprensión de los textos. Estudios realizados por Floyd y Carrell (1987) indican que estudiantes que leen en una segunda lengua y que no tienen suficiente conocimiento previo sobre los temas en particular, la pueden suplir a través de información relacionada con el conocimiento previo, que facilita la lectura de los estudiantes en dicha lengua.

Otras ayudas utilizadas que permitieron mejorar la comprensión de los textos multimodales en inglés fueron speed reading (22.4\%), ayuda ésta que en los registros de la interfaz aparece como la herramienta más usada y listening con (12.2\%), éstos son dos recursos que han sido utilizados el primero por Huang, Chern y Lin (2009, y el segundo por Al-Seghayer (2001), Sakar y Ercetin (2005), y Akyel y Ercetin (2009). Finalmente aparecen highlight (20.5\%), glossary (19.5\%), y key words (13.2\%).

Es importante resaltar que antes de que los estudiantes interactuaran con la segunda lectura mutimedial relacionada con los tsunamis, el profesor había realizado una modelación sobre el uso de las ayudas de manera eficiente, con la lectura que los estudiantes habían desarrollado en la sesión anterior y como resultado fue la única ocasión en que los estudiantes utilizaron todas las ayudas, es decir en un $100 \%$.

Lo que es acorde con lo que dice Hudson (2011), las estrategias deben ser enseñadas de forma explícita, con suficiente entrenamiento para que sean efectivas y deben ser utilizadas en diferentes contextos y con diversos tipos de textos. En este sentido los estudiantes necesitan saber utilizar las estrategias de forma automática antes de que ellos puedan monitorear con éxito la utilidad de las mismas.

Y finalmente se recomienda para mejorar el desempeño de los estudiantes en la lectura de textos en inglés en línea que los profesores que guían las practicas, hagan modelación y desarrollen una enseñanza explicita de las estrategias que se quieran utilizar como lo sugieren Huang, Chern y Lin (2009) y Coiro (2005).

\section{Conclusiones}

Las ayudas disponibles para la comprensión de textos multimodales en inglés tuvieron una gran acogida por parte de los estudiantes de ambos grupos, lo que se manifiesta en el $61 \%$ promedio general de uso de ayudas en las seis lecturas que se utilizaron para este estudio. Así mismo, el $78.5 \%$ de los estudiantes de ambos grupos declaró que aborda con más facilidad los textos multimodales que los impresos. A la par, la mayoría de los 
estudiantes de los dos grupos sugirió el uso de textos multimodales en los cursos regulares de competencia lectora en inglés.

En un cuestionario administrado a los estudiantes antes del inicio del proyecto, se encontró que la mayoría de los estudiantes de los dos grupos $65.6 \%$ tenían poco o muy poco el idioma inglés, se encontró también que el $81.3 \%$ de los estudiantes nunca o casi nunca leían textos en inglés en Internet. Basado en el análisis de la distribución de la media y la desviación estándar sobre el conocimiento del idioma inglés, se encontró que hubo aumentó estadísticamente significativo en el nivel de comprensión lectora alcanzado por los estudiantes del grupo uno, lo que quiere decir que la metodología empleada permitió por lo menos que un grupo de estudiantes con poco conocimiento del idioma inglés mejorara su nivel de comprensión en este idioma. De ahí que se pueda sugerir que este estudio, en pequeña medida, contribuye a disminuir la brecha entre estudiantes monolingües y bilingües en un mundo globalizado que cada vez requiere de personas que por lo menos puedan leer y comprender textos en el idioma inglés.

\section{Referencias}

Anderson, N.J. (2003). Scrolling, clicking and reading English: Online reading strategies in a second/foreign language. The Reading Matrix, 3(3), 1-33. Descargado el 13 de Marzo de 2009 desde http://www.readingmatrix.com/articles/anderson/article.pdf

Akyel, A., \& Ercetin, G. (2009). Hypermedia reading strategies employed by advanced learners of English. System 37, 136-152.

Al-Seghayer, K. (2001). The effect of multimedia annotation modes on L2 vocabulary acquisition: A comparative study. Language Learning and Technology, 5(1), 202-232.

Coiro, J. (2005). Making sense of online text, Educational Leadership 63 (2): 30-35.

Dörnyei, Z. (2007). Research methods in applied linguistics. Quantitative, qualitative and mixed methodologies. Oxford: Oxford University Press.

Floyd, P., \& Carrell, P. L. (1987). Effects on ESL reading of teaching cultural content schemata. Language Learning, 37, 89-108.

Gear, J., \& Gear, R. (2002). The computer-based TOEFL test, CD-ROM for computerbased exam, Cambridge preparation for the TOEFL test [CD-ROM], (3ra. Ed.). Cambridge: Cambridge University Press.

Grabe, W., \& Stoller, F. (2002). Teaching and researching reading. New York, NY: Pearson Education Limited.

Hernández, R., Fernández, C., \& Baptista, P. (2004). Metodología de la investigación. Toluca, Mexico: Mc Graw Hill.

Huang, H., Chern, C., \& Lin, C. (2009). EFL learners' use of online reading strategies and comprehension of texts: An exploratory study. Computers \& Education, 52(1), 13-26. 
Hudson, T. (2011). Teaching second language reading. Oxford: Oxford University Press.

Jiménez, J. (2009). Estudio a pequeña escala con EFL estudiantes universitarios utilizan ayudas para leer textos en un sitio web. Uni-pluri /versidad, Vol. 9 (3): 45-56.

Knobel, M., Coiro, J., Leu, D., \& Lankshear, C. (2008). Handbook of research on new literacies. New York, NY: Taylor \& Francis Group.

Leu, D.J. (2002). The new literacies: Research on reading instruction with the internet. En: A.E. Farstrup y S.J. Samuels (Eds.), What research has to say about reading instruction (pp. 310-336) Delaware: International Reading Association.

Mazzali-Lurati, S., \& Schulz, P. (2003). The actualization of reading strategies in hypermedia, Document Design 4(3), 246-268.

Perfetti, C. (1994). Psycholinguistics and reading ability. En M. A. Gernsbacher (Ed.), Handbook of psycholinguistics (pp. 849-894). San Diego, CA: Academic Press.

Plass, J., \& Jones, L. (2005). Multimedia learning in second language acquisition. Introduction to multimedia learning. En The Cambridge handbook of multimedia learning (pp. 467-488). New York, NY: Cambridge University Press.

Rodríguez, O. (2005). La triangulación como estrategia de investigación en ciencias sociales. $M I+D, 31$. Descargado el 25 de Septiembre de 2012 desde http://www.madrimasd.org/revista/revista31/tribuna/tribuna2.asp

Rubin, J., Chamot, A., Harris, V., \& Anderson, N. (2007). Intervening in the use of strategies. En Language Learner Strategies (pp.141-160). Oxford: Oxford University Press.

Sakar, A., \& Ercetin, G. (2005). Effectiveness of hypermedia annotations for foreign language reading. Journal of Computer Assisted Learning 21, 28-38.

Stakhnevich, J. (2002). Reading on the web: Implications for ESL Professionals. The Reading Matrix, 2(2), 1-30. Descargado el 13 de Enero de 2009 desde http://www.readingmatrix.com/articles/stakhnevich/article.pdf

Stanovich, K. E. (2000). Progress in understanding reading: Scientific foundations and new frontiers. New York: Guilford Press.

Zhang, S. \& Duke, N. (2008).Strategies for internet reading with different reading purposes: A descriptive study of twelve good internet readers. Journal of Literacy Research, 40(1): 128-162.

\footnotetext{
Referencias del autor:

John Jaime Jiménez Díaz es licenciado en Educación de la Universidad Autónoma Latinoamericana de Medellín; Especialista en Didáctica de lenguas extranjeras de la Universidad de Antioquia, Medellín; Magister en Didáctica del Inglés de la Universidad de Caldas, Manizales; Candidato a Doctor en Educación de la Universidad de Antioquia, Medellín, Colombia.

Email: jjjimenezd13@gmail.com

Para citar este artículo:

Jiménez, J. (2013). Ayudas en línea para lectura multimodal en lengua extranjera. Bellaterra Journal of Teaching \& Learning Language \& Literature, 6(2), 69-92.

${ }^{1}$ Esta investigación hace parte de la tesis doctoral del autor.

${ }^{2}$ Para tener una mejor comprensión de la interfaz y las lecturas, se puede acceder a ella en el sitio web: www.toreadenglish.com Nombre de usuario: invitado Contraseña: invitado.
} 
(Anexo 1)

Promedio de uso de ayudas según Grupo y Lectura

\begin{tabular}{|c|c|c|c|c|c|c|c|c|c|c|c|c|}
\hline Lectura & $\begin{array}{l}\text { Immi } \\
\text { to } \mathrm{Ar}\end{array}$ & $\begin{array}{l}\text { ation } \\
\text { erica }\end{array}$ & Tsu & amis & Vale & ine's & Schiz & renia & $\begin{array}{r}\mathrm{Imm} \\
\text { in }\end{array}$ & $\begin{array}{l}\text { ation } \\
\text { aly }\end{array}$ & Ove & veight \\
\hline Grupo & 1 & 2 & 1 & 2 & 1 & 2 & 1 & 2 & 1 & 2 & 1 & 2 \\
\hline Significancia & (A) & (B) & (A) & (B) & (A) & (B) & (A) & (B) & (A) & (B) & (A) & (B) \\
\hline $\begin{array}{l}\text { Veces que escuchó el texto } \\
\text { en inglés }\end{array}$ & $\begin{array}{l}1,8 \\
B\end{array}$ & 0,3 & 0,6 & 0,4 & 0,9 & 0,5 & 0,8 & 0,8 & 0,8 & 0,6 & 0,9 & 0,8 \\
\hline $\begin{array}{l}\text { Veces que escuchó el texto } \\
\text { en inglés más lento }\end{array}$ & $\begin{array}{l}1 \\
B\end{array}$ & 0,2 & 0,4 & 0,3 & 0,5 & 0,3 & 0,4 & 0,6 & 1,7 & 0,7 & 0,8 & 0,8 \\
\hline Veces que utilizó el traductor & 1,3 & 1,2 & 1 & 0,6 & 0,7 & 1,3 & 0,5 & $\begin{array}{c}1,4 \\
A\end{array}$ & 0,2 & 0,4 & 0,8 & 0,9 \\
\hline Veces que utilizó el glosario & 0,1 & 0,5 & 0,1 & 0,2 & 0,1 & $\begin{array}{c}0,5 \\
A\end{array}$ & 0,2 & 0,3 & 0,8 & 0,4 & 0,1 & 0,4 \\
\hline $\begin{array}{l}\text { Veces que requirió } \\
\text { información general sobre el } \\
\text { tema }\end{array}$ & 0 & $\begin{array}{c}0,5 \\
A\end{array}$ & 0,1 & 0,4 & 0,3 & 0,3 & 0,4 & 0,6 & 0,2 & 0,1 & 0,5 & 0,3 \\
\hline Veces que utilizó el mapa & 0 & 0 & 0 & 0 & 0 & 0 & 0 & 0 & 0 & 0 & 0 & 0 \\
\hline $\begin{array}{l}\text { Veces que utilizó el resaltador } \\
\text { de texto }\end{array}$ & $\begin{array}{c}0,8 \\
B\end{array}$ & 0,2 & $\begin{array}{c}1,5 \\
B\end{array}$ & 0,1 & 0,4 & 0,1 & 0,5 & 0,4 & $\begin{array}{c}0,5 \\
B\end{array}$ & 0 & 0,5 & 0,2 \\
\hline $\begin{array}{l}\text { Veces que requirió una } \\
\text { síntesis del parrafo }\end{array}$ & 0,2 & 0,5 & 0,1 & 0,4 & 0,2 & 0,3 & 0,4 & 0,6 & 0,5 & 0,2 & 0,2 & $\begin{array}{c}0,7 \\
A\end{array}$ \\
\hline $\begin{array}{l}\text { Veces que utilizó el } \\
\text { diccionario }\end{array}$ & 0,8 & 0,6 & 0,3 & 0,4 & 0,5 & 0,9 & 1,1 & 1,1 & $\begin{array}{c}0,9 \\
B\end{array}$ & 0,1 & 0,5 & 0,6 \\
\hline $\begin{array}{l}\text { Veces que necesitó conocer } \\
\text { las palabras claves y su } \\
\text { significado }\end{array}$ & 0,2 & 0,2 & 0,1 & $\begin{array}{c}0,7 \\
A\end{array}$ & 0,2 & 0,5 & 0,3 & 0,4 & 0,2 & 0,1 & 0,2 & 0,4 \\
\hline $\begin{array}{l}\text { Veces que requirió un } \\
\text { anticipo sobre el tema }\end{array}$ & 0,2 & 0,4 & 0,2 & 0,4 & 0,4 & 0,6 & 0,4 & 0,4 & 0,6 & 0,4 & 0,5 & 0,6 \\
\hline $\begin{array}{l}\text { Veces que requirió una síntesis } \\
\text { exhaustiva de cada uno de los } \\
\text { párrafos }\end{array}$ & 0 & 0,2 & 0,1 & 0,3 & 0,2 & 0,5 & 0,6 & 0,2 & 0,1 & 0 & 0,2 & 0,5 \\
\hline preguntas Buenas & 3,5 & $\stackrel{4,7}{A}$ & 4,6 & 3,9 & 4,4 & 3,7 & 4,4 & 4,9 & 6,5 & 5,9 & 5,3 & 4,8 \\
\hline
\end{tabular}

\section{(Anexo 2)}

Percepción de Uso de las ayudas según Lectura y Grupo

\begin{tabular}{|c|c|c|c|c|c|c|c|c|c|c|c|c|}
\hline \multirow{2}{*}{ LECTURA } & $\begin{array}{c}\text { Immigratio } \\
\text { n to } \\
\text { America }\end{array}$ & \multicolumn{2}{|c|}{$\begin{array}{c}\text { Tsunami } \\
\text { s }\end{array}$} & \multicolumn{2}{|c|}{$\begin{array}{c}\text { Valentine' } \\
\text { s day }\end{array}$} & \multicolumn{2}{|c|}{$\begin{array}{c}\text { Schizo- } \\
\text { phrenia }\end{array}$} & \multicolumn{2}{|c|}{$\begin{array}{c}\text { Immigratio } \\
\text { n in Italy }\end{array}$} & \multicolumn{2}{c|}{$\begin{array}{c}\text { Overweig } \\
\text { ht }\end{array}$} \\
\hline GRUPO & 1 & 2 & 1 & 2 & 1 & 2 & 1 & 2 & 1 & 2 & 1 & 2 \\
\hline SIGNIFICANCIA & (A) & (B) & (A) & (B) & (A) & (B) & (A) & (B) & (A) & (B) & (A) & (B) \\
\hline
\end{tabular}




\begin{tabular}{|c|c|c|c|c|c|c|c|c|c|c|c|c|c|}
\hline \multirow{2}{*}{$\begin{array}{c}\text { 1. Utilizó } \\
\text { todas las } \\
\text { ayudas } \\
\text { disponibles } \\
\text { para la } \\
\text { lectura }\end{array}$} & $\mathrm{Si}$ & 31.6 & 31.6 & 100 & 100 & $\begin{array}{c}11 . \\
8\end{array}$ & 15.8 & $\begin{array}{c}82 . \\
4\end{array}$ & $\begin{array}{c}82 . \\
4\end{array}$ & 61.9 & $\begin{array}{c}64 \\
3\end{array}$ & 88.2 & 85.7 \\
\hline & No & 68.4 & 68.4 & 0 & 0 & $\begin{array}{c}88 . \\
2\end{array}$ & 84.2 & $\begin{array}{c}17 . \\
6\end{array}$ & $\begin{array}{c}17 . \\
6\end{array}$ & 38.1 & $\begin{array}{c}35 . \\
7\end{array}$ & 11.8 & 14.3 \\
\hline \multirow{2}{*}{$\begin{array}{c}1 . \\
\text { Considera } \\
\text { que fue útil } \\
\text { el } \\
\text { instructivo } \\
\text { para } \\
\text { comprende } \\
\text { r el texto }\end{array}$} & $\mathrm{Si}$ & 0 & 0 & 0 & 0 & $\begin{array}{c}94 . \\
1\end{array}$ & 94.7 & 0 & 0 & 0 & 0 & 0 & 0 \\
\hline & No & 0 & 0 & 0 & 0 & 5.9 & 5.3 & 0 & 0 & 0 & 0 & 0 & 0 \\
\hline \multirow{12}{*}{$\begin{array}{l}\text { 2. Ayuda } \\
\text { que más } \\
\text { utilizó }\end{array}$} & Translator & 84.2 & 84.2 & $\begin{array}{c}64 . \\
7\end{array}$ & $\begin{array}{c}64 . \\
3\end{array}$ & $\begin{array}{c}76 . \\
5\end{array}$ & 78.9 & 100 & 100 & 57.1 & 100 & 82.4 & 85.7 \\
\hline & Dictionary & 73.7 & 73.7 & $\begin{array}{c}29 . \\
4\end{array}$ & $\begin{array}{c}28 . \\
6\end{array}$ & $\begin{array}{c}58 . \\
8\end{array}$ & 52.6 & $\begin{array}{c}17 . \\
6\end{array}$ & $\begin{array}{c}17 . \\
6\end{array}$ & $\begin{array}{c}42.9 \\
B\end{array}$ & 7.1 & 41.2 & 42.9 \\
\hline & $\begin{array}{l}\text { Speed } \\
\text { Reading }\end{array}$ & 21.1 & 21.1 & $\begin{array}{c}41 . \\
2\end{array}$ & $\begin{array}{c}42 . \\
9\end{array}$ & $\begin{array}{c}29 . \\
4\end{array}$ & 26.3 & 0 & 0 & 19 & $\begin{array}{c}35 . \\
7\end{array}$ & 17.6 & 21.4 \\
\hline & Highlight & 26.3 & 26.3 & $\begin{array}{c}47 . \\
1\end{array}$ & $\begin{array}{c}42 . \\
9\end{array}$ & 0 & 0 & $\begin{array}{c}17 . \\
6\end{array}$ & $\begin{array}{c}17 . \\
6\end{array}$ & 0 & $\begin{array}{c}14 . \\
3\end{array}$ & 35.3 & 28.6 \\
\hline & Glossary & 5.3 & 5.3 & 5.9 & 7.1 & $\begin{array}{c}23 . \\
5\end{array}$ & 26.3 & $\begin{array}{c}11 . \\
8\end{array}$ & $\begin{array}{c}11 . \\
8\end{array}$ & 33.3 & $\begin{array}{c}28 . \\
6\end{array}$ & 41.2 & 35.7 \\
\hline & Preview & 0 & 0 & 0 & 0 & $\begin{array}{c}35 . \\
3\end{array}$ & 36.8 & $\begin{array}{c}11 . \\
8\end{array}$ & $\begin{array}{c}11 . \\
8\end{array}$ & 23.8 & $\begin{array}{c}21 . \\
4\end{array}$ & 23.5 & 21.4 \\
\hline & Summary & 5.3 & 5.3 & 0 & 0 & $\begin{array}{c}17 . \\
6\end{array}$ & 15.8 & $\begin{array}{c}29 . \\
4\end{array}$ & $\begin{array}{c}29 . \\
4\end{array}$ & 0 & $\begin{array}{c}28 . \\
6\end{array}$ & 17.6 & 21.4 \\
\hline & Keywords & 5.3 & 5.3 & 0 & 0 & $\begin{array}{c}29 . \\
4\end{array}$ & 31.6 & 0 & 0 & 9.5 & 0 & 41.2 & 35.7 \\
\hline & $\begin{array}{l}\text { Background } \\
\text { Knowledge }\end{array}$ & 0 & 0 & 5.9 & 7.1 & $\begin{array}{c}29 . \\
4\end{array}$ & 31.6 & $\begin{array}{c}23 . \\
5\end{array}$ & $\begin{array}{c}23 . \\
5\end{array}$ & 4.8 & $\begin{array}{c}14 . \\
3\end{array}$ & 5.9 & 7.1 \\
\hline & Listening & 10.5 & 10.5 & $\begin{array}{c}17 . \\
6\end{array}$ & $\begin{array}{c}14 . \\
3\end{array}$ & 5.9 & 5.3 & $\begin{array}{c}11 . \\
8\end{array}$ & $\begin{array}{c}11 . \\
8\end{array}$ & 19 & $\begin{array}{c}14 . \\
3\end{array}$ & 11.8 & 14.3 \\
\hline & Outline & 0 & 0 & 0 & 0 & $\begin{array}{c}17 . \\
6\end{array}$ & 15.8 & 0 & 0 & 0 & 0 & 0 & 0 \\
\hline & Ninguna & 0 & 0 & 0 & 0 & 0 & 0 & 0 & 0 & 9.5 & 0 & 0 & 0 \\
\hline \multirow{12}{*}{$\begin{array}{l}\text { 3. Ayuda } \\
\text { que menos } \\
\text { utilizó }\end{array}$} & Listening & 73.7 & 73.7 & $\begin{array}{c}29 . \\
4\end{array}$ & $\begin{array}{c}28 . \\
6\end{array}$ & $\begin{array}{c}29 . \\
4\end{array}$ & 36.8 & $\begin{array}{c}58 . \\
8\end{array}$ & $\begin{array}{c}58 . \\
8\end{array}$ & 19 & $\begin{array}{c}28 . \\
6\end{array}$ & 41.2 & 42.9 \\
\hline & Highlight & 36.8 & 36.8 & $\begin{array}{c}35 . \\
3\end{array}$ & $\begin{array}{c}35 . \\
7\end{array}$ & $\begin{array}{c}47 . \\
1\end{array}$ & 47.4 & $\begin{array}{c}41 . \\
2\end{array}$ & $\begin{array}{c}41 . \\
2\end{array}$ & 33.3 & $\begin{array}{c}14 . \\
3\end{array}$ & 41.2 & 42.9 \\
\hline & Outline & 26.3 & 26.3 & $\begin{array}{c}70 . \\
6\end{array}$ & $\begin{array}{c}71 . \\
4\end{array}$ & $\begin{array}{c}17 . \\
6\end{array}$ & 15.8 & $\begin{array}{c}11 . \\
8\end{array}$ & $\begin{array}{c}11 . \\
8\end{array}$ & $\begin{array}{c}76.2 \\
\text { B }\end{array}$ & $\begin{array}{c}35 . \\
7\end{array}$ & 23.5 & 21.4 \\
\hline & Summary & 31.6 & 31.6 & $\begin{array}{c}52 . \\
9\end{array}$ & $\begin{array}{c}57 . \\
1\end{array}$ & $\begin{array}{c}41 . \\
2\end{array}$ & 36.8 & $\begin{array}{c}17 . \\
6\end{array}$ & $\begin{array}{c}17 . \\
6\end{array}$ & $\begin{array}{c}47.6 \\
B\end{array}$ & $\begin{array}{c}14 . \\
3\end{array}$ & 17.6 & 21.4 \\
\hline & Glossary & 26.3 & 26.3 & $\begin{array}{c}47 . \\
1\end{array}$ & $\begin{array}{c}42 . \\
9\end{array}$ & $\begin{array}{c}35 . \\
3\end{array}$ & 31.6 & $\begin{array}{c}29 . \\
4\end{array}$ & $\begin{array}{c}29 . \\
4\end{array}$ & $\begin{array}{c}38.1 \\
B\end{array}$ & 7.1 & 17.6 & 21.4 \\
\hline & $\begin{array}{l}\text { Background } \\
\text { Knowledge }\end{array}$ & 31.6 & 31.6 & $\begin{array}{c}23 . \\
5\end{array}$ & $\begin{array}{c}21 . \\
4\end{array}$ & $\begin{array}{c}41 . \\
2\end{array}$ & 36.8 & 0 & 0 & 61.9 & $\begin{array}{c}28 . \\
6\end{array}$ & 29.4 & 28.6 \\
\hline & Keywords & 21.1 & 21.1 & $\begin{array}{c}58 . \\
8\end{array}$ & $\begin{array}{c}57 . \\
1\end{array}$ & $\begin{array}{c}17 . \\
6\end{array}$ & 15.8 & 0 & 0 & 28.6 & 50 & 23.5 & 28.6 \\
\hline & $\begin{array}{l}\text { Speed } \\
\text { Reading }\end{array}$ & 21.1 & 21.1 & $\begin{array}{c}35 . \\
3\end{array}$ & $\begin{array}{c}35 . \\
7\end{array}$ & $\begin{array}{c}23 . \\
5\end{array}$ & 21.1 & 0 & 0 & 28.6 & $\begin{array}{c}35 . \\
7\end{array}$ & 35.3 & 35.7 \\
\hline & Dictionary & 10.5 & 10.5 & $\begin{array}{c}35 . \\
3\end{array}$ & $\begin{array}{c}42 . \\
9\end{array}$ & $\begin{array}{c}17 . \\
6\end{array}$ & 21.1 & $\begin{array}{c}47 . \\
1\end{array}$ & $\begin{array}{c}47 . \\
1\end{array}$ & 19 & 7.1 & 11.8 & 14.3 \\
\hline & Preview & 26.3 & 26.3 & $\begin{array}{c}11 . \\
8\end{array}$ & $\begin{array}{c}14 . \\
3\end{array}$ & $\begin{array}{c}23 . \\
5\end{array}$ & 21.1 & 0 & 0 & 19 & $\begin{array}{c}14 . \\
3\end{array}$ & 17.6 & 21.4 \\
\hline & Translator & 10.5 & 10.5 & 0 & 0 & $\begin{array}{c}17 . \\
6\end{array}$ & 15.8 & 0 & 0 & 9.5 & 0 & 0 & 0 \\
\hline & Ninguna & 0 & 0 & 0 & 0 & $\begin{array}{c}17 . \\
6\end{array}$ & 15.8 & 5.9 & 5.9 & 0 & 7.1 & 0 & 0 \\
\hline \multirow{2}{*}{$\begin{array}{l}\text { 4. Ayuda } \\
\text { que no } \\
\text { utilizó }\end{array}$} & Highlight & 47.4 & 47.4 & $\begin{array}{c}29 . \\
4\end{array}$ & $\begin{array}{c}28 . \\
6\end{array}$ & $\begin{array}{c}58 . \\
8\end{array}$ & 57.9 & $\begin{array}{c}58 . \\
8\end{array}$ & $\begin{array}{c}58 . \\
8\end{array}$ & 42.9 & $\begin{array}{c}42 . \\
9\end{array}$ & 35.3 & 42.9 \\
\hline & Outline & 36.8 & 36.8 & $\begin{array}{c}35 \\
3\end{array}$ & $\begin{array}{c}35 . \\
7\end{array}$ & $\begin{array}{c}41 . \\
2\end{array}$ & 42.1 & $\begin{array}{c}64 . \\
7\end{array}$ & $\begin{array}{c}64 . \\
7\end{array}$ & 47.6 & 50 & 41.2 & 42.9 \\
\hline
\end{tabular}




\begin{tabular}{|c|c|c|c|c|c|c|c|c|c|c|c|c|c|}
\hline & $\begin{array}{l}\text { Background } \\
\text { Knowledge }\end{array}$ & 31.6 & 31.6 & $\begin{array}{c}35 . \\
3\end{array}$ & $\begin{array}{c}35 . \\
7\end{array}$ & $\begin{array}{c}47 . \\
1\end{array}$ & 47.4 & $\begin{array}{c}64 . \\
7\end{array}$ & $\begin{array}{c}64 . \\
7\end{array}$ & 42.9 & $\begin{array}{c}42 . \\
9\end{array}$ & 41.2 & 35.7 \\
\hline & Keywords & 57.9 & 57.9 & $\begin{array}{c}11 . \\
8\end{array}$ & $\begin{array}{c}14 . \\
3\end{array}$ & $\begin{array}{c}35 . \\
3\end{array}$ & 36.8 & $\begin{array}{c}64 . \\
7\end{array}$ & $\begin{array}{c}64 . \\
7\end{array}$ & 47.6 & 50 & 5.9 & 7.1 \\
\hline & Preview & 31.6 & 31.6 & $\begin{array}{c}23 . \\
5\end{array}$ & $\begin{array}{c}28 . \\
6\end{array}$ & $\begin{array}{c}52 . \\
9\end{array}$ & 52.6 & $\begin{array}{c}47 . \\
1\end{array}$ & $\begin{array}{c}47 . \\
1\end{array}$ & 38.1 & 50 & 29.4 & 28.6 \\
\hline & Glossary & 47.4 & 47.4 & $\begin{array}{c}35 . \\
3\end{array}$ & $\begin{array}{c}35 . \\
7\end{array}$ & $\begin{array}{c}35 . \\
3\end{array}$ & 31.6 & $\begin{array}{c}35 . \\
3\end{array}$ & $\begin{array}{c}35 . \\
3\end{array}$ & 19 & $\begin{array}{c}35 . \\
7\end{array}$ & 29.4 & 28.6 \\
\hline & Listening & 26.3 & 26.3 & $\begin{array}{c}35 . \\
3\end{array}$ & $\begin{array}{c}28 . \\
6\end{array}$ & $\begin{array}{c}70 . \\
6\end{array}$ & 68.4 & $\begin{array}{c}29 . \\
4\end{array}$ & $\begin{array}{c}29 . \\
4\end{array}$ & 28.6 & $\begin{array}{c}14 . \\
3\end{array}$ & 23.5 & 21.4 \\
\hline & Summary & 31.6 & 31.6 & $\begin{array}{c}17 . \\
6\end{array}$ & $\begin{array}{c}21 . \\
4\end{array}$ & $\begin{array}{c}29 . \\
4\end{array}$ & 31.6 & $\begin{array}{c}35 . \\
3\end{array}$ & $\begin{array}{c}35 . \\
3\end{array}$ & $\begin{array}{c}61.9 \\
B\end{array}$ & $\begin{array}{c}14 . \\
3\end{array}$ & 23.5 & 21.4 \\
\hline & $\begin{array}{l}\text { Speed } \\
\text { Reading }\end{array}$ & 15.8 & 15.8 & $\begin{array}{c}29 . \\
4\end{array}$ & $\begin{array}{c}35 . \\
7\end{array}$ & $\begin{array}{c}35 . \\
3\end{array}$ & 36.8 & $\begin{array}{c}47 . \\
1\end{array}$ & $\begin{array}{c}47 . \\
1\end{array}$ & 42.9 & $\begin{array}{c}14 . \\
3\end{array}$ & 17.6 & 21.4 \\
\hline & Dictionary & 5.3 & 5.3 & $\begin{array}{c}11 . \\
8\end{array}$ & $\begin{array}{c}14 . \\
3\end{array}$ & 5.9 & 5.3 & $\begin{array}{c}29 . \\
4\end{array}$ & $\begin{array}{c}29 . \\
4\end{array}$ & 19 & $\begin{array}{c}64 . \\
3 \\
\text { A }\end{array}$ & 23.5 & 21.4 \\
\hline & Translator & 21.1 & 21.1 & $\begin{array}{c}17 . \\
6\end{array}$ & $\begin{array}{c}21 . \\
4\end{array}$ & $\begin{array}{c}23 . \\
5\end{array}$ & 21.1 & 0 & 0 & 28.6 & 0 & 0 & 0 \\
\hline & Ninguna & 5.3 & 5.3 & 0 & 0 & $\begin{array}{c}11 . \\
8\end{array}$ & 10.5 & 5.9 & 5.9 & 14.3 & 7.1 & 5.9 & 7.1 \\
\hline \multirow{11}{*}{$\begin{array}{l}\text { 5. Ayudas } \\
\text { que le } \\
\text { permitieron } \\
\text { una mejor } \\
\text { comprensió } \\
\text { n del texto }\end{array}$} & Translator & 78.9 & 78.9 & $\begin{array}{c}64 . \\
7\end{array}$ & $\begin{array}{c}64 . \\
3\end{array}$ & $\begin{array}{c}82 . \\
4\end{array}$ & 84.2 & $\begin{array}{c}70 . \\
6\end{array}$ & $\begin{array}{c}70 . \\
6\end{array}$ & 57.1 & 100 & 88.2 & 85.7 \\
\hline & Dictionary & 47.4 & 47.4 & $\begin{array}{c}17 . \\
6\end{array}$ & $\begin{array}{c}14 . \\
3\end{array}$ & $\begin{array}{c}70 . \\
6\end{array}$ & 68.4 & $\begin{array}{c}11 . \\
8\end{array}$ & $\begin{array}{c}11 . \\
8\end{array}$ & 47.6 & 0 & 23.5 & 28.6 \\
\hline & Listening & 10.5 & 10.5 & $\begin{array}{c}52 . \\
9\end{array}$ & 50 & 5.9 & 5.3 & $\begin{array}{c}17 . \\
6\end{array}$ & $\begin{array}{c}17 . \\
6\end{array}$ & 4.8 & 0 & 29.4 & 28.6 \\
\hline & Preview & 0 & 0 & $\begin{array}{c}17 . \\
6\end{array}$ & $\begin{array}{c}21 . \\
4\end{array}$ & $\begin{array}{c}47 . \\
1\end{array}$ & 42.1 & $\begin{array}{c}11 . \\
8\end{array}$ & $\begin{array}{c}11 . \\
8\end{array}$ & 14.3 & $\begin{array}{c}14 . \\
3\end{array}$ & 5.9 & 7.1 \\
\hline & Glossary & 21.1 & 21.1 & 0 & 0 & $\begin{array}{c}29 . \\
4\end{array}$ & 31.6 & 0 & 0 & 28.6 & 0 & 17.6 & 14.3 \\
\hline & Summary & 0 & 0 & $\begin{array}{c}17 . \\
6\end{array}$ & $\begin{array}{c}21 . \\
4\end{array}$ & $\begin{array}{c}17 . \\
6\end{array}$ & 15.8 & $\begin{array}{c}17 . \\
6\end{array}$ & $\begin{array}{c}17 . \\
6\end{array}$ & 0 & $\begin{array}{c}14 . \\
3\end{array}$ & 17.6 & 21.4 \\
\hline & $\begin{array}{l}\text { Speed } \\
\text { Reading }\end{array}$ & 10.5 & 10.5 & 5.9 & 7.1 & $\begin{array}{c}23 . \\
5\end{array}$ & 21.1 & $\begin{array}{c}11 . \\
8\end{array}$ & $\begin{array}{c}11 . \\
8\end{array}$ & 14.3 & 7.1 & 11.8 & 14.3 \\
\hline & $\begin{array}{l}\text { Background } \\
\text { Knowledge }\end{array}$ & 0 & 0 & 5.9 & 7.1 & $\begin{array}{c}29 . \\
4\end{array}$ & 26.3 & $\begin{array}{c}23 . \\
5\end{array}$ & $\begin{array}{c}23 . \\
5\end{array}$ & 0 & $\begin{array}{c}14 . \\
3\end{array}$ & 0 & 0 \\
\hline & Highlight & 0 & 0 & $\begin{array}{c}17 . \\
6\end{array}$ & $\begin{array}{c}14 . \\
3\end{array}$ & 0 & 0 & 0 & 0 & 9.5 & 0 & 47.1 & 42.9 \\
\hline & Outline & 0 & 0 & $\begin{array}{c}17 . \\
6\end{array}$ & $\begin{array}{c}21 . \\
4\end{array}$ & $\begin{array}{c}17 . \\
6\end{array}$ & 15.8 & 0 & 0 & 0 & 7.1 & 5.9 & 7.1 \\
\hline & Keywords & 5.3 & 5.3 & 0 & 0 & 5.9 & 5.3 & 5.9 & 5.9 & 4.8 & 0 & 23.5 & 21.4 \\
\hline \multirow{12}{*}{$\begin{array}{l}\text { 6. Ayudas } \\
\text { que utilizó, } \\
\text { pero no } \\
\text { fueron } \\
\text { útiles para } \\
\text { mejorar la } \\
\text { comprensió } \\
\text { n del texto }\end{array}$} & Ninguna & 36.8 & 36.8 & 5.9 & 7.1 & $\begin{array}{c}41 . \\
2\end{array}$ & 42.1 & $\begin{array}{c}52 . \\
9\end{array}$ & $\begin{array}{c}52 . \\
9\end{array}$ & 33.3 & $\begin{array}{c}42 . \\
9\end{array}$ & 35.3 & 42.9 \\
\hline & Highlight & 36.8 & 36.8 & 0 & 0 & $\begin{array}{c}11 . \\
8\end{array}$ & 10.5 & $\begin{array}{c}17 . \\
6\end{array}$ & $\begin{array}{c}17 . \\
6\end{array}$ & 28.6 & 0 & 0 & 0 \\
\hline & Listening & 10.5 & 10.5 & $\begin{array}{c}17 . \\
6\end{array}$ & $\begin{array}{c}14 . \\
3\end{array}$ & $\begin{array}{c}11 . \\
8\end{array}$ & 10.5 & $\begin{array}{c}17 . \\
6\end{array}$ & $\begin{array}{c}17 . \\
6\end{array}$ & 0 & $\begin{array}{c}28 . \\
6\end{array}$ & 17.6 & 14.3 \\
\hline & Glossary & 10.5 & 10.5 & 5.9 & 7.1 & $\begin{array}{c}17 . \\
6\end{array}$ & 15.8 & $\begin{array}{c}11 . \\
8\end{array}$ & $\begin{array}{c}11 . \\
8\end{array}$ & 0 & 0 & 17.6 & 14.3 \\
\hline & $\begin{array}{l}\text { Background } \\
\text { Knowledge }\end{array}$ & 15.8 & 15.8 & 0 & 0 & 5.9 & 5.3 & 0 & 0 & 28.6 & 0 & 5.9 & 7.1 \\
\hline & Keywords & 0 & 0 & $\begin{array}{c}17 . \\
6\end{array}$ & $\begin{array}{c}21 . \\
4\end{array}$ & $\begin{array}{c}11 . \\
8\end{array}$ & 10.5 & 0 & 0 & 0 & $\begin{array}{c}21 . \\
4\end{array}$ & 5.9 & 7.1 \\
\hline & Summary & 0 & 0 & 0 & 0 & 5.9 & 10.5 & $\begin{array}{c}11 . \\
8\end{array}$ & $\begin{array}{c}11 . \\
8\end{array}$ & 14.3 & 0 & 11.8 & 14.3 \\
\hline & Translator & 0 & 0 & $\begin{array}{c}29 . \\
4\end{array}$ & $\begin{array}{c}28 . \\
6\end{array}$ & 0 & 0 & 0 & 0 & 9.5 & 0 & 0 & 0 \\
\hline & $\begin{array}{l}\text { Speed } \\
\text { Reading }\end{array}$ & 0 & 0 & $\begin{array}{c}11 . \\
8\end{array}$ & 7.1 & 5.9 & 5.3 & 0 & 0 & 4.8 & 0 & 17.6 & 14.3 \\
\hline & Outline & 10.5 & 10.5 & $\begin{array}{c}11 . \\
8\end{array}$ & $\begin{array}{c}14 . \\
3\end{array}$ & 0 & 0 & 0 & 0 & 4.8 & 7.1 & 0 & 0 \\
\hline & Preview & 5.3 & 5.3 & 0 & 0 & 0 & 0 & 0 & 0 & 0 & 0 & 0 & 0 \\
\hline & Dictionary & 0 & 0 & 0 & 0 & 5.9 & 5.3 & 0 & 0 & 0 & 0 & 0 & 0 \\
\hline \multirow{2}{*}{$\begin{array}{l}\text { 7. Califique } \\
\text { el nivel de }\end{array}$} & 2 & 31.6 & 31.6 & 0 & 0 & 0 & 0 & 0 & 0 & 0 & 0 & 0 & 0 \\
\hline & 3 & 21.1 & 21.1 & 0 & 0 & 0 & 0 & 0 & 0 & 0 & 0 & 0 & 0 \\
\hline
\end{tabular}




\begin{tabular}{|c|c|c|c|c|c|c|c|c|c|c|c|c|c|}
\hline $\begin{array}{l}\text { dificultad } \\
\text { del test de } \\
\text { comprensió } \\
\text { n del texto } \\
\text { Immigration } \\
\text { to America }\end{array}$ & 4 & 47.4 & 47.4 & 0 & 0 & 0 & 0 & 0 & 0 & 0 & 0 & 0 & 0 \\
\hline \multirow{7}{*}{$\begin{array}{l}\text { 7. Cuál test } \\
\text { de } \\
\text { comprensió } \\
\text { n fue más } \\
\text { difícil }\end{array}$} & $\begin{array}{l}\text { Schizophren } \\
\text { ia }\end{array}$ & 0 & 0 & 0 & 0 & 0 & 0 & $\begin{array}{c}35 . \\
3\end{array}$ & $\begin{array}{c}35 . \\
3\end{array}$ & $\begin{array}{c}95.2 \\
\text { B }\end{array}$ & $\begin{array}{c}71 . \\
4\end{array}$ & 0 & 0 \\
\hline & $\begin{array}{l}\text { Valentine's } \\
\text { day }\end{array}$ & 0 & 0 & 0 & 0 & $\begin{array}{c}41 . \\
2\end{array}$ & 47.4 & $\begin{array}{c}47 . \\
1\end{array}$ & $\begin{array}{c}47 . \\
1\end{array}$ & 0 & 0 & 0 & 0 \\
\hline & $\begin{array}{l}\text { Immigration } \\
\text { to America }\end{array}$ & 0 & 0 & $\begin{array}{c}82 . \\
4\end{array}$ & $\begin{array}{c}78 . \\
6\end{array}$ & 0 & 0 & 0 & 0 & 0 & 0 & 17.6 & 21.4 \\
\hline & Tsunamis & 0 & 0 & $\begin{array}{c}29 . \\
4\end{array}$ & $\begin{array}{c}35 . \\
7\end{array}$ & $\begin{array}{c}47 . \\
1\end{array}$ & 42.1 & 0 & 0 & 0 & 0 & 0 & 0 \\
\hline & Overweight & 0 & 0 & 0 & 0 & 0 & 0 & 0 & 0 & 0 & 0 & 70.6 & 64.3 \\
\hline & $\begin{array}{l}\text { No } \\
\text { Responde }\end{array}$ & 0 & 0 & 0 & 0 & $\begin{array}{c}11 . \\
8\end{array}$ & 10.5 & $\begin{array}{c}17 . \\
6\end{array}$ & $\begin{array}{c}17 . \\
6\end{array}$ & 4.8 & $\begin{array}{c}14 . \\
3\end{array}$ & 5.9 & 7.1 \\
\hline & $\begin{array}{l}\text { Immigration } \\
\text { in Italy }\end{array}$ & 0 & 0 & 0 & 0 & 0 & 0 & 0 & 0 & 0 & $\begin{array}{c}42 . \\
9\end{array}$ & 5.9 & 7.1 \\
\hline \multirow{7}{*}{$\begin{array}{l}\text { 8. Cuáles } \\
\text { ayudas } \\
\text { utilizó para } \\
\text { responder } \\
\text { las } \\
\text { preguntas } \\
\text { del test } \\
\text { sobre la } \\
\text { lectura }\end{array}$} & Translator & 0 & 0 & 100 & 100 & $\begin{array}{c}85 . \\
7\end{array}$ & 81.3 & 100 & 100 & 66.7 & 0 & 93.8 & 92.3 \\
\hline & Dictionary & 0 & 0 & $\begin{array}{c}23 . \\
1\end{array}$ & $\begin{array}{c}18 . \\
2\end{array}$ & $\begin{array}{c}35 . \\
7 \\
\end{array}$ & 37.5 & $\begin{array}{c}35 . \\
3\end{array}$ & $\begin{array}{c}35 . \\
3\end{array}$ & 33.3 & 0 & 18.8 & 15.4 \\
\hline & Summary & 0 & 0 & 0 & 0 & 0 & 0 & $\begin{array}{c}17 . \\
6\end{array}$ & $\begin{array}{c}17 . \\
6\end{array}$ & 0 & 0 & 0 & 0 \\
\hline & Glossary & 0 & 0 & 0 & 0 & $\begin{array}{c}14 . \\
3\end{array}$ & 12.5 & 0 & 0 & 13.3 & 0 & 0 & 0 \\
\hline & Highlight & 0 & 0 & 0 & 0 & 0 & 0 & 0 & 0 & 0 & 0 & 18.8 & 15.4 \\
\hline & $\begin{array}{l}\text { Background } \\
\text { Knowledge }\end{array}$ & 0 & 0 & 0 & 0 & 0 & 0 & $\begin{array}{c}11 . \\
8\end{array}$ & $\begin{array}{c}11 . \\
8\end{array}$ & 0 & 0 & 0 & 0 \\
\hline & Preview & 0 & 0 & 0 & 0 & 7.1 & 6.3 & 0 & 0 & 0 & 0 & 0 & 0 \\
\hline \multirow{3}{*}{$\begin{array}{c}\text { 10. Cuál } \\
\text { tipo de } \\
\text { texto } \\
\text { aborda con } \\
\text { más } \\
\text { facilidad ( } \\
\text { multimodal } \\
\text { es o } \\
\text { impresos) }\end{array}$} & $\begin{array}{l}\text { multimodale } \\
\mathrm{s}\end{array}$ & 68.4 & 68.4 & $\begin{array}{c}82 . \\
4\end{array}$ & $\begin{array}{c}85 . \\
7\end{array}$ & $\begin{array}{c}82 . \\
4\end{array}$ & 84.2 & $\begin{array}{c}64 . \\
7\end{array}$ & $\begin{array}{c}64 . \\
7\end{array}$ & 100 & $\begin{array}{c}85 . \\
7\end{array}$ & 76.5 & 78.6 \\
\hline & Impresos & 31.6 & 31.6 & $\begin{array}{c}17 . \\
6\end{array}$ & $\begin{array}{c}14 . \\
3\end{array}$ & $\begin{array}{c}17 . \\
6\end{array}$ & 15.8 & $\begin{array}{c}17 . \\
6\end{array}$ & $\begin{array}{c}17 . \\
6\end{array}$ & 0 & $\begin{array}{c}14 . \\
3\end{array}$ & 23.5 & 21.4 \\
\hline & $\begin{array}{l}\text { No } \\
\text { Responde }\end{array}$ & 0 & 0 & 0 & 0 & 0 & 0 & $\begin{array}{c}17 . \\
6\end{array}$ & $\begin{array}{c}17 . \\
6\end{array}$ & 0 & 0 & 0 & 0 \\
\hline \multirow{2}{*}{$\begin{array}{l}\text { 10. Cuál } \\
\text { tipo de } \\
\text { texto lee y } \\
\text { comprende } \\
\text { mejor }\end{array}$} & $\begin{array}{l}\text { multimodale } \\
\mathrm{s}\end{array}$ & 0 & 0 & $\begin{array}{c}82 . \\
4\end{array}$ & $\begin{array}{c}85 . \\
7\end{array}$ & 0 & 0 & 0 & 0 & 0 & 0 & 0 & 0 \\
\hline & Impresos & 0 & 0 & $\begin{array}{c}17 . \\
6\end{array}$ & $\begin{array}{c}14 . \\
3\end{array}$ & 0 & 0 & 0 & 0 & 0 & 0 & 0 & 0 \\
\hline \multirow{4}{*}{$\begin{array}{l}\text { 11. Cuál de } \\
\text { los } \\
\text { siguientes } \\
\text { tipos de } \\
\text { textos le } \\
\text { gustaría } \\
\text { continuar } \\
\text { leyendo en } \\
\text { este curso }\end{array}$} & $\begin{array}{l}\text { Textos } \\
\text { multimodale } \\
\text { s en inglés } \\
\text { como el que } \\
\text { acabas de } \\
\text { leer }\end{array}$ & 36.8 & 36.8 & $\begin{array}{c}17 \\
6\end{array}$ & $\begin{array}{c}21 . \\
4\end{array}$ & $\begin{array}{c}52 . \\
9\end{array}$ & 52.6 & 0 & 0 & $\begin{array}{c}85.7 \\
B\end{array}$ & $\begin{array}{c}21 . \\
4\end{array}$ & 0 & 0 \\
\hline & $\begin{array}{l}\text { Textos } \\
\text { impresos en } \\
\text { inglés }\end{array}$ & 15.8 & 15.8 & $\begin{array}{c}11 . \\
8\end{array}$ & $\begin{array}{c}14 . \\
3\end{array}$ & 0 & 0 & $\begin{array}{c}17 . \\
6\end{array}$ & $\begin{array}{c}17 . \\
6\end{array}$ & 0 & 0 & 0 & 0 \\
\hline & $\begin{array}{l}\text { Combinar } \\
\text { textos } \\
\text { multimodale } \\
\text { s e } \\
\text { impresos }\end{array}$ & 31.6 & 31.6 & $\begin{array}{c}70 . \\
6\end{array}$ & $\begin{array}{c}64 . \\
3\end{array}$ & $\begin{array}{c}47 . \\
1\end{array}$ & 47.4 & $\begin{array}{c}82 . \\
4\end{array}$ & $\begin{array}{c}82 . \\
4\end{array}$ & 14.3 & $\begin{array}{c}71 . \\
4 \\
\text { A }\end{array}$ & 100 & 100 \\
\hline & $\begin{array}{l}\text { No } \\
\text { Responde }\end{array}$ & 15.8 & 15.8 & 0 & 0 & 0 & 0 & 0 & 0 & 0 & 7.1 & 0 & 0 \\
\hline \multirow{2}{*}{$\begin{array}{l}\text { 12. Le } \\
\text { gustaría } \\
\text { continuar }\end{array}$} & $\begin{array}{l}\text { No } \\
\text { Responde }\end{array}$ & 15.8 & 15.8 & 0 & 0 & 0 & 0 & 0 & 0 & 0 & 0 & 0 & 0 \\
\hline & $\mathrm{Si}$ & 15.8 & 15.8 & 0 & 0 & 0 & 0 & 0 & 0 & 0 & 0 & 0 & 0 \\
\hline
\end{tabular}




\begin{tabular}{|c|c|c|c|c|c|c|c|c|c|c|c|c|c|}
\hline $\begin{array}{c}\text { leyendo en } \\
\text { este curso } \\
\text { textos } \\
\text { impresos } \\
\text { en inglés }\end{array}$ & No & 68.4 & 68.4 & 0 & 0 & 0 & 0 & 0 & 0 & 0 & 0 & 0 & 0 \\
\hline \multirow[b]{2}{*}{$\begin{array}{c}13 . \\
\text { Sugeriría } \\
\text { usted el } \\
\text { uso de } \\
\text { textos } \\
\text { multimodal } \\
\text { es en los } \\
\text { cursos de } \\
\text { competenci } \\
\text { a lectora }\end{array}$} & Si & 26.3 & 26.3 & $\begin{array}{c}64 . \\
7\end{array}$ & $\begin{array}{c}64 . \\
3\end{array}$ & $\begin{array}{c}58 . \\
8\end{array}$ & 57.9 & $\begin{array}{c}64 . \\
7\end{array}$ & $\begin{array}{c}64 . \\
7\end{array}$ & 28.6 & 50 & 17.6 & 21.4 \\
\hline & No & 73.7 & 73.7 & $\begin{array}{c}35 . \\
3\end{array}$ & $\begin{array}{c}35 . \\
7\end{array}$ & $\begin{array}{c}41 . \\
2\end{array}$ & 42.1 & $\begin{array}{c}35 . \\
3\end{array}$ & $\begin{array}{c}35 . \\
3\end{array}$ & 71.4 & 50 & 82.4 & 78.6 \\
\hline \multirow{2}{*}{$\begin{array}{l}\text { 14. Quiere } \\
\text { comentar } \\
\text { algo } \\
\text { adicional } \\
\text { sobre su } \\
\text { experiencia }\end{array}$} & $\mathrm{Si}$ & 31.6 & 31.6 & $\begin{array}{c}29 . \\
4\end{array}$ & $\begin{array}{c}28 . \\
6\end{array}$ & $\begin{array}{c}41 . \\
2\end{array}$ & 42.1 & $\begin{array}{c}23 . \\
5\end{array}$ & $\begin{array}{c}23 . \\
5\end{array}$ & 0 & $\begin{array}{c}42 . \\
9\end{array}$ & 11.8 & 14.3 \\
\hline & No & 68.4 & 68.4 & $\begin{array}{c}70 . \\
6\end{array}$ & $\begin{array}{c}71 . \\
4\end{array}$ & $\begin{array}{c}58 . \\
8\end{array}$ & 57.9 & $\begin{array}{c}76 . \\
5\end{array}$ & $\begin{array}{c}76 . \\
5\end{array}$ & 100 & $\begin{array}{c}57 . \\
1\end{array}$ & 88.2 & 85.7 \\
\hline
\end{tabular}

\section{(Anexo 3)}

Promedio de uso de ayudas Total

\begin{tabular}{|c|c|c|c|c|c|c|c|c|c|}
\hline & & & & & & & ctura & & \\
\hline & $\begin{array}{l}\text { Tot } \\
\text { al }\end{array}$ & $\begin{array}{c}\text { Grup } \\
01\end{array}$ & $\begin{array}{c}\text { Grup } \\
02\end{array}$ & $\begin{array}{l}\text { Immigrati } \\
\text { on to } \\
\text { America }\end{array}$ & $\begin{array}{c}\text { Tsunam } \\
\text { is }\end{array}$ & $\begin{array}{l}\text { Valentine } \\
\text { 's day }\end{array}$ & $\begin{array}{l}\text { Schizophre } \\
\text { nia }\end{array}$ & $\begin{array}{l}\text { Immigrati } \\
\text { on in Italy }\end{array}$ & $\begin{array}{c}\text { Overweig } \\
\text { ht }\end{array}$ \\
\hline $\begin{array}{c}\text { Significan } \\
\text { cia }\end{array}$ & & (A) & (B) & (A) & (B) & (C) & (D) & (E) & (F) \\
\hline $\begin{array}{l}\text { Cantidad } \\
\text { de } \\
\text { Preguntas } \\
\text { Buenas }\end{array}$ & 4,6 & 4,7 & 4,6 & 4 & 4,2 & 4,1 & 4,6 & $\begin{array}{c}6,2 \\
A B C D F\end{array}$ & $\begin{array}{c}5,1 \\
A B C\end{array}$ \\
\hline $\begin{array}{l}\text { Veces que } \\
\text { escuchó el } \\
\text { texto en } \\
\text { inglés }\end{array}$ & 0,8 & $\begin{array}{l}1 \\
B\end{array}$ & 0,5 & $\begin{array}{c}1,1 \\
B\end{array}$ & 0,5 & 0,7 & 0,8 & 0,7 & 0,8 \\
\hline $\begin{array}{l}\text { Veces que } \\
\text { escuchó el } \\
\text { texto en } \\
\text { inglés más } \\
\text { lento }\end{array}$ & 0,6 & 0,8 & 0,5 & 0,6 & 0,4 & 0,4 & 0,5 & $\begin{array}{l}1,3 \\
\text { B C }\end{array}$ & 0,8 \\
\hline $\begin{array}{l}\text { Veces que } \\
\text { utilizó el } \\
\text { traductor }\end{array}$ & 0,9 & 0,8 & 1 & $\begin{array}{c}1,2 \\
E\end{array}$ & 0,8 & $\begin{array}{c}0,9 \\
E\end{array}$ & $\begin{array}{c}0,9 \\
E\end{array}$ & 0,3 & $\begin{array}{l}0,9 \\
\mathrm{E}\end{array}$ \\
\hline $\begin{array}{l}\text { Veces que } \\
\text { utilizó el } \\
\text { glosario }\end{array}$ & 0,3 & 0,2 & 0,4 & 0,3 & 0,1 & 0,3 & 0,3 & $\begin{array}{c}0,6 \\
A B C D F\end{array}$ & 0,2 \\
\hline $\begin{array}{l}\text { Veces que } \\
\text { requirió } \\
\text { informació } \\
\text { n general } \\
\text { sobre el } \\
\text { tema }\end{array}$ & 0,3 & 0,3 & 0,4 & 0,2 & 0,3 & 0,3 & $\begin{array}{c}0,5 \\
E\end{array}$ & 0,2 & 0,4 \\
\hline $\begin{array}{l}\text { Veces que } \\
\text { utilizó el } \\
\text { mapa }\end{array}$ & 0,0 & 0 & 0 & 0 & $\begin{array}{l}0 \\
.\end{array}$ & 0 & 0 & 0 & 0 \\
\hline $\begin{array}{l}\text { Veces que } \\
\text { utilizó el } \\
\text { resaltador } \\
\text { de texto }\end{array}$ & 0,5 & $\begin{array}{l}0,7 \\
B\end{array}$ & 0,2 & 0,6 & $\begin{array}{l}0,8 \\
C E\end{array}$ & 0,3 & 0,5 & 0,3 & 0,4 \\
\hline
\end{tabular}




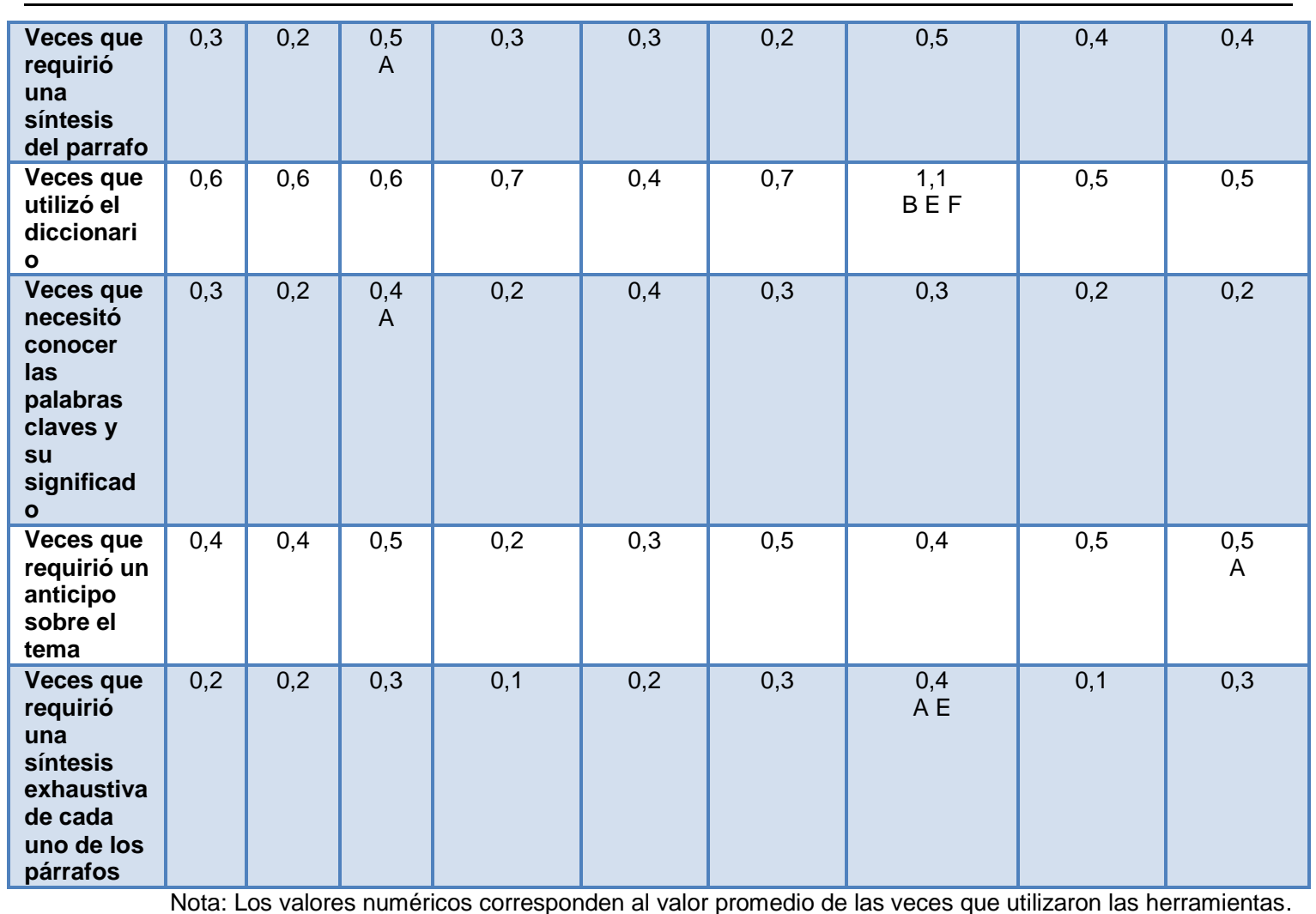

(Anexo 4)

Evaluación metodológica

\begin{tabular}{|c|c|c|c|c|c|}
\hline \multirow{2}{*}{ Etapa } & \multirow{2}{*}{ Ítem } & \multirow{2}{*}{ Respuesta } & Grupo 1 & Grupo 2 & Total \\
\hline & & & $(n=17)$ & $(n=15)$ & $(n=32)$ \\
\hline \multirow{17}{*}{$\begin{array}{l}\text { Evaluación } \\
\text { Multimedia }\end{array}$} & \multirow{5}{*}{$\begin{array}{l}\text { El diseño es atractivo, } \\
\text { agradable y estético }\end{array}$} & Totalmente en desacuerdo & 0 & , 0 & 0 \\
\hline & & En desacuerdo & ,0 & 1,0 & ,4 \\
\hline & & De acuerdo & 32,8 & 27,0 & 30,3 \\
\hline & & Totalmente de acuerdo & 57,8 & 70,0 & 63,2 \\
\hline & & No Responde & 9,4 & 2,0 & 6,1 \\
\hline & \multirow{5}{*}{$\begin{array}{l}\text { Los gráficos y animaciones } \\
\text { son pertinentes y ayudan a } \\
\text { mejorar su proceso de } \\
\text { comprensión lectora }\end{array}$} & Totalmente en desacuerdo & 2,3 & 0 & 1,3 \\
\hline & & En desacuerdo & 10,9 & 0 & 6,1 \\
\hline & & De acuerdo & 44,5 & 41,0 & 43,0 \\
\hline & & Totalmente de acuerdo & 32,0 & 57,0 & 43,0 \\
\hline & & No Responde & 10,2 & 2,0 & 6,6 \\
\hline & \multirow{5}{*}{$\begin{array}{l}\text { Los íconos son adecuados } \\
\text { para la función que } \\
\text { representan }\end{array}$} & Totalmente en desacuerdo & 0 & 1,0 & 4 \\
\hline & & En desacuerdo & 8,6 & 1,0 & 5,3 \\
\hline & & De acuerdo & 47,7 & 37,0 & 43,0 \\
\hline & & Totalmente de acuerdo & 34,4 & 59,0 & 45,2 \\
\hline & & No Responde & 9,4 & 2,0 & 6,1 \\
\hline & \multirow{2}{*}{$\begin{array}{l}\text { La navegación y } \\
\text { exploración del material }\end{array}$} & Totalmente en desacuerdo & ,8 & 0 & ,4 \\
\hline & & En desacuerdo & 1,6 & 2,0 & 1,8 \\
\hline
\end{tabular}




\begin{tabular}{|c|c|c|c|c|c|}
\hline & \multirow[t]{3}{*}{ multimodal es fácil } & De acuerdo & 48,4 & 37,0 & 43,4 \\
\hline & & Totalmente de acuerdo & 39,8 & 59,0 & 48,2 \\
\hline & & No Responde & 9,4 & 2,0 & 6,1 \\
\hline \multirow{20}{*}{$\begin{array}{l}\text { Evaluación Sobre } \\
\text { el Tema }\end{array}$} & \multirow{5}{*}{$\begin{array}{l}\text { Son coherentes con el } \\
\text { propósito del curso }\end{array}$} & Totalmente en desacuerdo & 0 & 0 & 0 \\
\hline & & En desacuerdo & 5,5 & , 0 & 3,1 \\
\hline & & De acuerdo & 38,3 & 34,0 & 36,4 \\
\hline & & Totalmente de acuerdo & 44,5 & 63,0 & 52,6 \\
\hline & & No Responde & 11,7 & 3,0 & 7,9 \\
\hline & \multirow{5}{*}{$\begin{array}{l}\text { Son pertinentes (los temas } \\
\text { tratados son los que se } \\
\text { requieren en el curso) }\end{array}$} & Totalmente en desacuerdo & 0 & 0 &, 0 \\
\hline & & En desacuerdo & 7,8 & ,0 & 4,4 \\
\hline & & De acuerdo & 44,5 & 32,0 & 39,0 \\
\hline & & Totalmente de acuerdo & 35,9 & 65,0 & 48,7 \\
\hline & & No Responde & 11,7 & 3,0 & 7,9 \\
\hline & \multirow{5}{*}{$\begin{array}{l}\text { Están distribuidos y } \\
\text { dosificados } \\
\text { adecuadamente }\end{array}$} & Totalmente en desacuerdo & 0 & , 0 & 0 \\
\hline & & En desacuerdo & 1,6 & 1,0 & 1,3 \\
\hline & & De acuerdo & 46,9 & 38,0 & 43,0 \\
\hline & & Totalmente de acuerdo & 39,8 & 58,0 & 47,8 \\
\hline & & No Responde & 11,7 & 3,0 & 7,9 \\
\hline & \multirow{5}{*}{$\begin{array}{l}\text { Posibilitan una mejor } \\
\text { comprensión lectora }\end{array}$} & Totalmente en desacuerdo & 0 & 0 & 0 \\
\hline & & En desacuerdo & 4,7 & 1,0 & 3,1 \\
\hline & & De acuerdo & 41,4 & 37,0 & 39,5 \\
\hline & & Totalmente de acuerdo & 42,2 & 59,0 & 49,6 \\
\hline & & No Responde & 11,7 & 3,0 & 7,9 \\
\hline \multirow{20}{*}{$\begin{array}{l}\text { Evaluación del } \\
\text { Profesor }\end{array}$} & \multirow{5}{*}{$\begin{array}{l}\text { Motiva y despierta interés } \\
\text { por el curso }\end{array}$} & Totalmente en desacuerdo & 1,6 & 0 & 9 \\
\hline & & En desacuerdo & , 0 & 0 & 0 \\
\hline & & De acuerdo & 43,8 & 28,0 & 36,8 \\
\hline & & Totalmente de acuerdo & 43,0 & 70,0 & 54,8 \\
\hline & & No Responde & 11,7 & 2,0 & 7,5 \\
\hline & \multirow[t]{5}{*}{ Conoce y domina los temas } & Totalmente en desacuerdo & 1,6 &, 0 & ,9 \\
\hline & & En desacuerdo & 3,9 &, 0 & 2,2 \\
\hline & & De acuerdo & 34,4 & 24,0 & 29,8 \\
\hline & & Totalmente de acuerdo & 48,4 & 74,0 & 59,6 \\
\hline & & No Responde & 11,7 & 2,0 & 7,5 \\
\hline & \multirow{5}{*}{$\begin{array}{l}\text { Retroalimenta } \\
\text { adecuadamente el } \\
\text { desarrollo de las } \\
\text { actividades }\end{array}$} & Totalmente en desacuerdo & 1,6 & 0 & 9 \\
\hline & & En desacuerdo & 1,6 &, 0 & ,9 \\
\hline & & De acuerdo & 48,4 & 38,0 & 43,9 \\
\hline & & Totalmente de acuerdo & 36,7 & 60,0 & 46,9 \\
\hline & & No Responde & 11,7 & 2,0 & 7,5 \\
\hline & \multirow{5}{*}{$\begin{array}{l}\text { Es claro y preciso en las } \\
\text { respuestas, sugerencias e } \\
\text { instrucciones }\end{array}$} & Totalmente en desacuerdo & 1,6 & 0 & 9 \\
\hline & & En desacuerdo & 5,5 & 1,0 & 3,5 \\
\hline & & De acuerdo & 44,5 & 37,0 & 41,2 \\
\hline & & Totalmente de acuerdo & 36,7 & 60,0 & 46,9 \\
\hline & & No Responde & 11,7 & 2,0 & 7,5 \\
\hline Autoevaluación & Logró fortalecer su & $\mathrm{Si}$ & 82,0 & 93,0 & 86,8 \\
\hline
\end{tabular}




\begin{tabular}{|c|c|c|c|c|}
\hline \multirow{2}{*}{$\begin{array}{l}\text { comprensión lectora con el } \\
\text { uso de la multimedia }\end{array}$} & No & 6,3 & 5,0 & 5,7 \\
\hline & No Responde & 11,7 & 2,0 & 7,5 \\
\hline \multirow{3}{*}{$\begin{array}{l}\text { Tuvo dificultades para el } \\
\text { desarrollo de la } \\
\text { comprensión lectora a partir } \\
\text { del uso de la multimedia }\end{array}$} & $\mathrm{Si}$ & 39,8 & 40,0 & 39,9 \\
\hline & No & 48,4 & 58,0 & 52,6 \\
\hline & No Responde & 11,7 & 2,0 & 7,5 \\
\hline \multirow{3}{*}{$\begin{array}{l}\text { Considera que la } \\
\text { multimedia realmente le } \\
\text { aporta a su proceso de } \\
\text { comprensión lectora e } \\
\text { lengua extranjera }\end{array}$} & $\mathrm{Si}$ & 82,8 & 97,0 & 89,0 \\
\hline & No & 5,5 & 1,0 & 3,5 \\
\hline & No Responde & 11,7 & 2,0 & 7,5 \\
\hline
\end{tabular}

